Cahiers de la recherche sur les droits fondamentaux

$7 \mid 2009$

L'universalisme des droits en question(s). La

Déclaration universelle des Droits de l'homme, 60 ans après

\title{
La destinée universaliste des droits culturels : les articles 22 et 27 de la Déclaration universelle des Droits de l'homme
}

Olivia Bui-Xuan

\section{(2) OpenEdition \\ Journals}

\section{Édition électronique}

URL : https://journals.openedition.org/crdf/6702

DOI : $10.4000 /$ crdf. 6702

ISSN : 2264-1246

Éditeur

Presses universitaires de Caen

\section{Édition imprimée}

Date de publication : 31 décembre 2009

Pagination : 133-142

ISBN : 978-2-84133-342-4

ISSN : $1634-8842$

Référence électronique

Olivia Bui-Xuan, « La destinée universaliste des droits culturels : les articles 22 et 27 de la Déclaration universelle des Droits de l'homme », Cahiers de la recherche sur les droits fondamentaux [En ligne], 7| 2009, mis en ligne le 15 décembre 2020, consulté le 14 novembre 2022. URL : http:// journals.openedition.org/crdf/6702 ; DOI : https://doi.org/10.4000/crdf.6702 


\title{
La destinée universaliste des droits culturels: les articles 22 et 27 de la Déclaration universelle des Droits de l'homme
}

\author{
Olivia BUI-XUAN \\ Maître de conférences en droit public à l'Université d'Évry-Val-d'Essonne
}

I. Des droits à la culture aux droits des cultures

A. Les droits culturels comme droits à la culture

1. Le droit d'accéder à la culture

2. Les droits d'auteur

B. Les droits culturels comme droits des cultures

II. Les droits culturels : des droits individuels aux droits des groupes

A. Des droits individuels aux droits collectifs

B. Des droits collectifs aux droits des communautés culturelles

La question de l'universalité des Droits de l'homme se pose avec d'autant plus d'acuité que l'on s'intéresse aux droits culturels. Définis de façon floue en 1948, ces droits relèvent d'un domaine oscillant entre l'universel et le particulier, selon la définition de la culture que l'on adopte. En effet, si la culture est synonyme d'expression artistique et littéraire, on peut a priori concevoir des droits culturels pour tous les individus; si elle est appréhendée de façon plus large au sens de civilisation, les droits culturels risquent de devenir des droits particularistes.

Or, en soixante ans, souvent présentés comme des droits secondaires, comme les parents pauvres de la catégorie plus vaste "droits économiques, sociaux et culturels ${ }^{1}$, les droits culturels ont fait l'objet d'importantes mutations. Afin qu'ils puissent être exercés par tous les êtres humains, afin qu'ils aient donc une portée universaliste effective, leur définition a subi une double évolution: à l'origine plutôt conçus comme des droits individuels à la culture, concept purement occidental ${ }^{2}$, ils sont de plus en plus entendus comme les droits collectifs des cultures. Il est pourtant permis de se demander si ce glissement sémantique à visée universaliste ne contient pas intrinsèquement un relativisme culturel difficilement compatible avec l'universalisme visé.

1. Voir, par exemple, J. Symonides, «Les droits culturels: une catégorie négligée de Droits de l'homme », Revue internationale des sciences sociales, $\mathrm{n}^{\circ}$ 158, décembre 1998, p. 619.

2. Glen Johnson indique que «le cadre de référence européen a dominé de façon écrasante les délibérations qui ont donné naissance à la Déclaration universelle» (G. Johnson, «La rédaction de la Déclaration universelle (1946-1948) », La Déclaration universelle des Droits de l'homme-40 anniversaire, Paris, L'Harmattan, 1988, p. 62) ; sur la question de savoir si les Droits de l'homme relèvent eux-mêmes d'une culture particulière, voir R. Panikkar, « La

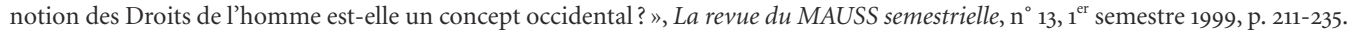




\section{Des droits à la culture aux droits des cultures}

Dès que l'on évoque les droits culturels, on se heurte à un problème d'interprétation. Que recouvre exactement cette notion? Il existe en effet une ambiguïté intrinsèque au mot «culture" à laquelle la Déclaration universelle des Droits de l'homme (DUDH) n'a pas mis fin. Boris Martin distingue ainsi la «culture-bénéfice définie comme "l'enrichissement de l'esprit par des exercices intellectuels" » de la "culture-identité » ${ }^{3}$. Par suite, les droits culturels peuvent être compris comme des droits à la culture (culture-bénéfice) ou comme des droits des cultures (cultures-identités).

Le fait de ne pas avoir clarifié dès le départ la notion de droits culturels est problématique car, selon l'acception adoptée, les enjeux, au regard de l'universalisme, sont différents: si par droits culturels, on entend droits à la culture, la compatibilité avec l'universalisme semble, au premier abord, possible; en revanche, si on conçoit les droits culturels comme les droits des cultures, cette compatibilité est moins évidente. Alors qu'en 1948, la première acception était privilégiée, depuis une vingtaine d'années, le droit international opte plutôt pour la seconde.

\section{A. Les droits culturels comme droits à la culture}

À l'époque de la rédaction de la DUDH, les droits culturels recouvrent incontestablement les droits à la culture, lesquels comportent deux volets : le droit d'accéder à la culture et les droits d'auteur.

\section{Le droit d'accéder à la culture}

En vertu de l'article 22 de la DUDH, « Toute personne [...] est fondée à obtenir la satisfaction des droits économiques, sociaux et culturels indispensables à sa dignité et au libre développement de sa personnalité, grâce à l'effort national et à la coopération internationale, compte tenu de l'organisation et des ressources de chaque pays. »

Bien qu'imprécis, ces droits culturels, associés aux droits économiques et sociaux, se comprennent comme des droits à la culture, entendue comme l' « ensemble des connaissances acquises qui permettent de développer le sens critique, le goût, le jugement ${ }^{4}$ (arts, littérature, cinéma, etc.). Ces droits culturels s'opposent ainsi à la censure, à l'obscurantisme; ils relèvent finalement de la liberté d'expression et participent de la liberté d'opinion, mais vont au-delà puisque, droits-créances, ils exigent une prestation des États.
De tels droits culturels sont donc a priori contraignants pour les États, lesquels se voient dicter une obligation de faire - et pas simplement une obligation d'abstention. Celle-ci est pourtant, dans le même temps, fortement atténuée: d'une part, ce sont seulement les droits culturels « indispensables à [1] a dignité et au libre développement de [1]a personnalité » des individus que les États sont censés garantir ; d'autre part, les États semblent exonérés d'une obligation de résultat dans la mesure où l'obligation est subordonnée à «l'organisation et aux ressources du pays ». Ainsi, dans les États aux ressources insuffisantes, ces droits culturels, tous nécessaires à l'émancipation des hommes qu'ils soient, peuvent être extrêmement réduits, voire inexistants. Les termes mêmes de l'article 22 semblent donc ôter toute sa portée à cette disposition: dans la mesure où ce sont les États euxmêmes qui estiment qu'un droit culturel est indispensable à la dignité d'un individu et que l'organisation et les ressources d'un pays permettent leur développement, cet article n'a en fait guère de portée normative.

De surcroît, aucune précision quant aux contours de ces droits culturels n'est apportée par cette disposition. Toutefois, au regard de l'article 27 de la DUDH, ils semblent plutôt recouvrir un droit à la culture-bénéfice : l'alinéa $1^{\mathrm{er}}$ de cet article dispose en effet que «Toute personne a le droit de prendre part librement à la vie culturelle de la communauté, de jouir des arts et de participer au progrès scientifique et aux bienfaits qui en résultent ». Nul ne peut donc être exclu de la vie culturelle. Comme l'explique Gérard Cohen-Jonathan, cette disposition « est une réponse directe à l'ordonnance de Goebbels qui interdisait aux juifs de participer aux manifestations de la culture allemande (12 novembre 1938) et à celle sur la confiscation des œuvres d'art "dégénéré" (3 mai 1938) »" Combiné avec la disposition de l'article 22 de la Déclaration, ce droit-abstention - en vertu duquel l'État ne doit pas entraver la liberté des individus d'accéder et de participer à la vie culturelle et de se livrer à une activité créatrice - se comprend aussi comme un droit-créance : les États ont l'obligation de mettre la culture à la portée de tous, autrement dit, pour employer une expression à la mode, ils doivent "démocratiser la culture », préoccupation hautement louable, mais purement occidentale, qui peut être prolongée par un service public culturel.

Ainsi, Janusz Symonides indique-t-il que «la conférence intergouvernementale sur les politiques culturelles convoquées par l'Unesco en 1970 a conclu que l'article 27 de la Déclaration universelle implique que les autorités qui ont la charge d'une communauté ont le devoir de fournir à tous ses membres les moyens effectifs de participer à la vie culturelle, et non pas seulement celui de respecter leur droit à y prendre part $»^{6}$.

\footnotetext{
3. B. Martin, «Les droits culturels comme mode d'interprétation et de mise en œuvre des Droits de l'homme», La revue du MAUSS semestrielle, ${ }^{\circ} 13$, $1^{\text {er }}$ semestre 1999 , p. 237.

4. Le Petit Robert.

5. G. Cohen-Jonathan, "Sciences et culture», La Déclaration universelle des Droits de l'homme, textes rassemblés par M. Bettati, O. Duhamel et L. Greislamer, Paris, Gallimard (Folio), 1998, p. 140.

6. J. Symonides, L'Unesco et la Déclaration universelle des Droits de l'homme, Paris, L'Harmattan - Unesco, 1991, p. 166. Il précise: «En ce qui concerne les efforts qu'il conviendrait que les États membres ou les autorités compétentes entreprennent pour permettre à tous les individus de participer pleinement
} 
Le lien établi entre culture et progrès scientifique n'est pas évident, cette dernière notion étant de surcroît ignorée par certaines civilisations. Il est d'ailleurs permis de se demander s'il n'aurait pas été plus logique de la faire figurer à l'article 26 consacré au droit à l'éducation. Il s'agit en effet, pour les États membres, d'encourager les échanges entre scientifiques, lesquels doivent également pouvoir publier et diffuser les résultats de leurs recherches.

Par ailleurs, même si la DUDH n'en fait pas mention, les droits culturels conçus comme des droits d'accéder à la culture comprennent également le droit de jouir du patrimoine culturel : la protection du patrimoine a rapidement été régie par des conventions internationales au premier rang desquelles la Convention pour la protection des biens culturels en cas de conflit armé de 1954, la Convention concernant les mesures à prendre pour interdire et empêcher l'importation, l'exportation et le transfert de propriété illicites des biens culturels de 1970 et la Convention concernant la protection du patrimoine mondial, culturel et naturel de 1972.

Parallèlement au droit d'accéder à la culture, les droits culturels se déclinent sous une autre forme, liée aux droits de la propriété littéraire et artistique.

\section{Les droits d'auteur}

Le 2 alinéa de l'article 27 dispose que «Chacun a droit à la protection des intérêts moraux et matériels découlant de toute production scientifique, littéraire ou artistique dont il est l'auteur ». Cette disposition a fait l'objet de controverses lors de la rédaction de la Déclaration: alors que les membres de la Commission estimaient qu'un tel droit n'avait pas sa place dans la DUDH, René Cassin a insisté pour que cet alinéa y figure ${ }^{7}$. Là encore, les droits d'auteur, le droit à la protection des intérêts découlant d'une production, déjà consacrés par l'article 13 de la Déclaration américaine des droits et devoirs de l'homme de mars 1948, procèdent d'une conception occidentale de la culture: dans certaines sociétés, une telle notion est inenvisageable et même incompréhensible, les productions intellectuelles ne donnant lieu à aucune appropriation particulière. Pourtant, dès 1952, cette disposition a trouvé un prolongement dans la Convention universelle sur le droit de l'auteur, texte révisé en 1971.

Qu'il s'agisse du droit d'accéder à la culture ou du droit à la propriété littéraire et artistique, on constate que les deux déclinaisons originelles des droits culturels sont présentes à l'article 15 du Pacte international relatif aux droits économiques, sociaux et culturels de 1966. Celuici dispose en effet: «1. Les États parties au présent Pacte reconnaissent à chacun le droit: a. de participer à la vie culturelle; b. de bénéficier du progrès scientifique et de ses applications; c. de bénéficier de la protection des intérêts moraux et matériels découlant de toute production scientifique, littéraire ou artistique dont il est l'auteur. Aucun droit-créance n'est cependant associé à ces droits culturels.»

Dans leur première acception, les droits culturels consacrés par la DUDH relevaient ainsi assez largement d'une conception ethno-centriste de la culture : de nombreux États pouvaient difficilement les mettre en œuvre, soit que certaines notions leur soient étrangères, soit qu’ils manquent de moyens pour les garantir. De surcroît, en dépit du principe d'indivisibilité des Droits de l'homme, de tels droits pouvaient passer pour secondaires. Aussi, dans un contexte d'affirmation de l'égale dignité de toutes les cultures, afin que les droits culturels soient garantis à tous les individus, quelle que soit leur civilisation, un autre sens a-t-il progressivement été ajouté : la culture a été appréhendée de façon anthropologique, comme une «culture-identité »; les droits culturels ont ainsi été liés aux identités culturelles. Cette évolution a permis de donner de l'importance à la culture: au risque de dissocier une culture essentielle et une culture qui serait synonyme de faste, la culture est devenue un élément intrinsèque de l'identité qu'il est indispensable de préserver; au rebours de la «culture-bénéfice», cette conception avait par ailleurs le mérite de pouvoir être défendue par toutes les sociétés.

Si, dans les années 1960, les deux sens du terme «culture» semblent d'abord se superposer, les dernières évolutions des textes internationaux montrent que l'on a subrepticement assisté à une substitution de l'acception «culture-identité » à celle de "culture-bénéfice»: dans les conventions et déclarations internationales les plus récentes, les droits culturels sont entendus presque exclusivement comme les droits des cultures, au sens de droits à la préservation des identités culturelles. Ce faisant, c'est rien de moins que le droit à la différence culturelle qui est consacré.

\section{B. Les droits culturels comme droits des cultures}

Le glissement sémantique de droits à la culture à droits des cultures ${ }^{8}$ peut s'expliquer de différentes manières. D'une part, à partir des années 1960, les minorités ont progressivement été prises en compte par le droit international: face à la crainte que la mondialisation n'uniformise les cultures - entendues cette fois au sens anthropologique comme l'" ensemble des aspects intellectuels propres à une civilisation, une nation ${ }^{9}$ - est

et librement à la création de la culture et à ses bienfaits, la Recommandation [concernant la participation et la contribution des masses populaires à la vie culturelle, élaborée par la Conférence générale en 1976] souligne la nécessité de démocratiser les moyens et les instruments de l'action culturelle. »

7. M. Agi, «L'action personnelle de René Cassin », in La Déclaration universelle des Droits de l'homme, 1948-1998. Avenir d'un idéal commun, Commission nationale consultative des Droits de l'homme (éd.), Paris, La Documentation française, 1999, p. 171.

8. Pour certains auteurs, le fait que les droits culturels soient conçus comme les droits des cultures ne pose aucun problème. Voir, par exemple, A. Fenet, «Droits culturels et communauté mondiale», in L'homme, ses territoires, ses cultures. Mélanges offerts à André-Hubert Mesnard, J. Fialaire et É. Mondielli (dir.), Paris, LGDJ, 2006, p. 247-263; J.-M. Pontier, «Entre le local, le national et le supranational : les droits culturels », AJDA, 2000, p. $50-57$. 9. Le Petit Robert. 
apparue une volonté de protéger les différentes identités culturelles, notamment celles des cultures minoritaires. D'autre part, ce mouvement a franchi un nouveau palier après la chute du mur de Berlin. Enfin, les événements du 11 septembre 2001 ont accéléré la dynamique : des travaux de l'Unesco, il ressort la conviction que la lutte contre le terrorisme doit passer par la reconnaissance et la valorisation de la « la diversité culturelle». La culture a ainsi été appréhendée de plus en plus largement comme intégrant, au-delà des aspects intellectuels et artistiques d'une société, tous ses aspects anthropologiques, comme la langue, les traditions, les modes de vie.

Ce glissement peut paraître d'autant plus discret que, dans les textes internationaux, droits à la culture et droits des cultures sont le plus souvent confondus: ils s'entremêlent, entretenant la confusion quant à l'acception à donner à l'adjectif «culturel». La mutation a lieu dès 1966 à l'article 27 du Pacte international relatif aux droits civils et politiques, article dans lequel la notion de " vie culturelle », déjà présente à l'alinéa $1^{\text {er }}$ de l'article 27 de la DUDH, est pour la première fois étroitement liée à celle de "minorités». Cet article dispose que «dans les États où il existe des minorités ethniques, religieuses ou linguistiques, les personnes appartenant à ces minorités ne peuvent être privées du droit d'avoir, en commun avec les autres membres de leur groupe, leur propre vie culturelle». Alors qu'initialement le droit à la vie culturelle consacré à l'article 27 de la DUDH semblait être déconnecté du concept de minorité, on assiste ici à un changement de sens, l'adjectif «culturel» étant appréhendé au sens de "civilisation ». De surcroît, cette disposition particularise le terme «culture» : en affirmant que les personnes appartenant à des minorités ont droit à leur "propre» vie culturelle, elle sous-entend qu'au sein d'un même État, peuvent coexister différentes cultures (au sens de civilisation), et que les cultures minoritaires doivent être préservées face à la culture majoritaire. Le fait d'appréhender les droits culturels à travers le prisme minoritaire se confirme à la lecture de l'article 2 de la Déclaration des droits des personnes appartenant à des minorités nationales ou ethniques, religieuses et linguistiques de 1992, en vertu duquel « 1 . Les personnes appartenant à des minorités nationales ou ethniques, religieuses et linguistiques (ci-après dénommées personnes appartenant à des minorités) ont le droit de jouir de leur propre culture, de professer et de pratiquer leur propre religion et d'utiliser leur propre langue, en privé et en public, librement et sans ingérence ni discrimination quelconque. 2. Les personnes appartenant à des minorités ont le droit de participer pleinement à la vie culturelle, religieuse, sociale, économique et publique $[. .$.$] .»$
La Déclaration universelle de l'Unesco sur la diversité culturelle de 2001 entretient l'ambiguïté quant au sens à donner au terme "culture». En effet, elle lie, dès l'article $1^{\text {er }}$, culture et «pluralité des identités », l'expression «identité culturelle» étant omniprésente dans le texte. La «culture-bénéfice» est ainsi constamment entremêlée à la «culture-identité». Pour preuve, en vertu de l'article 5, "Toute personne doit [...] pouvoir s'exprimer, créer et diffuser ses œuvres dans la langue de son choix et en particulier dans sa langue maternelle; [...] toute personne doit pouvoir participer à la vie culturelle de son choix et exercer ses propres pratiques culturelles ».

Le glissement sémantique autant que la confusion établie entre les différents sens du mot «culture» franchit ici un palier qualitatif: si le fait de mêler, dans une même disposition, «œuvres», «vie culturelle» et «pratiques culturelles » peut sembler anodin, l'expression « pratiques culturelles » est susceptible de désigner des traditions totalement déconnectées des "pratiques artistiques», comme l'obligation pour les femmes de cacher leur visage ou d'être excisées. Ce changement de sens est d'autant plus important que le préambule indique que «La culture doit être considérée comme l'ensemble de traits distinctifs spirituels et matériels, intellectuels et affectifs qui caractérisent une société ou un groupe social ; elle englobe, outre les arts et les lettres, les modes de vie, les façons de vivre ensemble, les systèmes de valeurs, les traditions et les croyances" $"$

La Convention de l'Unesco sur la protection et la promotion de la diversité des expressions culturelles de 2005 consacre pleinement l'élargissement de l'acception du terme «culture» et a le mérite de tenter de la clarifier : la culture n'est définitivement plus seulement considérée sous le seul angle des beaux-arts et des belles lettres. À ce titre, l'Unesco reconnaît elle-même quatre paliers dans l'évolution du terme culture: 1. dans les années 1950 et 1960, la culture - au départ entendue comme production artistique - est élargie au concept d'identité culturelle, notamment dans le contexte de la décolonisation; 2. dans les années 1970 et 1980, un lien est fait entre culture et développement; 3. dans les années 1980 et 1990, la prise en compte de l'identité culturelle s'étend à toutes les personnes exclues, qu'il s'agisse des personnes appartenant à une minorité, à un peuple autochtone ou des personnes immigrées ; 4. dans les années 1990 et 2000 est recherché un dialogue des cultures dont la diversité est présentée comme une richesse $^{10}$. En dépit de ces précisions, la notion de «culture» est plus que jamais ambiguë dans la Convention de l'Unesco de 2005 , le terme étant paradoxalement employé tantôt au sens de «culture-bénéfice ${ }^{11}$, tantôt

10. Voir la présentation faite par l'Unesco de la Convention sur la protection et la promotion de la diversité des expressions culturelles, disponible sur son site Internet, p. 2 et 3. Elle précise : «En écho à la définition élargie de la notion de culture, la Déclaration universelle de l’Unesco sur la diversité culturelle aborde le double défi de la diversité culturelle: d'une part, assurer une interaction harmonieuse et un vouloir vivre ensemble entre personnes et groupes aux identités culturelles plurielles, variées et dynamiques; d'autre part, défendre une diversité créatrice, à savoir la multiplicité des formes par lesquelles les cultures révèlent leurs expressions patrimoniales et contemporaines à travers le temps et l'espace. » Nous soulignons.

11. Par exemple, dans le préambule, est reconnue «l'importance des droits de propriété intellectuelle pour soutenir les personnes qui participent à la créativité culturelle». 
en tant que «culture-identité » ${ }^{12}$. La tentative de définition de la diversité culturelle ${ }^{13}$ illustre bien cette confusion; l'article 4 indique que : "La diversité culturelle se manifeste non seulement dans les formes variées à travers lesquelles le patrimoine culturel de l'humanité est exprimé, enrichi et transmis grâce à la variété des expressions culturelles, mais aussi à travers divers modes de création artistique, de production, de diffusion, de distribution et de jouissance des expressions culturelles, quels que soient les moyens et les technologies utilisés »; de même les «politiques et mesures culturelles» renvoient «aux politiques et mesures relatives à la culture, à un niveau local, national, régional ou international, qu'elles soient centrées sur la culture en tant que telle (sic), ou destinées à avoir un effet direct sur les expressions culturelles des individus, groupes ou sociétés, y compris sur la création, la production, la diffusion et la distribution d'activités, de biens ou de services culturels et sur l'accès à ceux-ci ». La «culture en tant que telle» est donc disjointe de la «culture» telle que définie dans le préambule, lequel rappelle par ailleurs que «la diversité linguistique est un élément fondamental de la diversité culturelle » et considère «l'importance de la vitalité des cultures pour tous, $y$ compris pour les personnes appartenant aux minorités et pour les peuples autochtones, telle qu'elle se manifeste par leur liberté de créer, diffuser et distribuer leurs expressions culturelles traditionnelles et d'y avoir accès de manière à favoriser leur propre développement». Ainsi, la question de savoir si la culture est une marchandise comme les autres masque-t-elle une pluralité d'acceptions de ce terme: parfois synonyme de "diversité ethnique ", la diversité culturelle n'est plus uniquement conçue comme un prolongement de la problématique relative à l'exception culturelle.

Enfin, même si elle n'a qu'une valeur symbolique, la Déclaration de Fribourg relative aux droits culturels adoptée le 7 mai $2007^{14}$ confirme l'évolution du sens donné aux droits culturels, ces derniers ne pouvant se concevoir qu'en lien avec les identités. Ainsi, aux termes de l'article $2 \mathrm{a}$, « Le terme "culture" recouvre les valeurs, les croyances, les convictions, les langues, les savoirs et les arts, les traditions, institutions et modes de vie par lesquels une personne ou un groupe exprime son humanité et les significations qu'il donne à son existence et à son développement ».

Il s'agit donc clairement d'une acception anthropologique dont les arts ne constituent qu'une composante parmi d'autres. L'article 5, consacré plus spécifiquement au «droit d'accéder et de participer librement [...] à la vie culturelle à travers les activités de son choix [...] comprend notamment: la liberté de s'exprimer, en public ou en privé dans la, ou les, langues de son choix; la liberté d'exercer, en accord avec les droits reconnus dans la présente Déclaration, ses propres pratiques culturelles et de poursuivre un mode de vie associé à la valorisation de ses ressources culturelles, notamment dans le domaine de l'utilisation, de la production et de la diffusion de biens et de services ; la liberté de développer et de partager des connaissances, des expressions culturelles, de conduire des recherches et de participer aux différentes formes de création ainsi qu'à leurs bienfaits ; le droit à la protection des intérêts moraux et matériels liés aux œuvres qui sont le fruit de son activité culturelle».

Cette disposition est tout à fait significative du brouillage effectué entre droit à la culture et droit des cultures, le second prévalant, voire absorbant désormais le premier. On a ainsi l'impression que les droits des cultures ont en quelque sorte été substitués aux droits à la culture, comme si les premiers pouvaient compenser l'ineffectivité des droits culturels-créances.

Finalement, au vu de ces différents textes, un constat s'impose: les droits culturels (y compris les droits à la culture) sont désormais inséparables des identités culturelles; tout se passe comme si ces droits étaient aujourd'hui d'abord conçus pour les personnes appartenant à des minorités, voire leur étaient réservés. Certes, les droits d'une culture peuvent participer au droit à la culture: diffuser la littérature rédigée dans une langue minoritaire relève par exemple des deux déclinaisons des droits culturels. En ce sens, la reconnaissance des droits des cultures est nécessaire pour parachever l'universalisme du droit à la culture, initialement conçu comme un droit occidental. Mais, dans le même temps, les droits d'une culture peuvent aussi heurter les Droits de l'homme, dans la mesure où ils sont susceptibles de reléguer l'individu au second plan par rapport au groupe d'appartenance.

\section{Les droits culturels: des droits individuels aux droits des groupes}

Pour René Cassin, l'universalité des Droits de l'homme contenus dans la DUDH était intimement liée à l'être humain. Selon lui, la Déclaration « est universelle par son inspiration, par son expression, par son contenu, par son champ d'application, par son potentiel, et elle proclame directement les droits de l'être humain au regard de tous autres, à quelques groupes sociaux auxquels ils appartiennent les uns et les autres ${ }^{15}$.

12. Par exemple, dans le préambule, est reconnue «l'importance des savoirs traditionnels en tant que source de richesse immatérielle et matérielle, et en particulier des systèmes de connaissance des peuples autochtones, et leur contribution positive au développement durable, ainsi que la nécessité d'assurer leur protection et promotion de façon adéquate».

13. Sur cette notion, voir G. Gagné (dir.), La diversité culturelle. Vers une convention internationale effective?, Québec, Éditions Fides, 2005. D’autres textes, comme la Déclaration du Conseil de l'Europe sur la diversité culturelle de 2000 ou, dans le cadre de l'Organisation internationale de la francophonie, la Déclaration de Cotonou sur la diversité culturelle de 2001, se réferent à cette notion.

14. Ce texte a été rédigé par un groupe de travail de l'Institut interdisciplinaire d'éthique et des Droits de l'homme de l'Université de Fribourg qui, en lien avec l'Unesco, mène depuis plusieurs années une réflexion sur les droits culturels. Voir P. Meyer-Bisch (dir.), Les droits culturels. Projet de déclaration, Paris - Fribourg, Unesco - Éditions universitaires, 1998.

15. R. Cassin, «L'homme sujet de droit international et la protection universelle de l'homme », in Mélanges Georges Scelle. La technique et les principes du droit public, Paris, LGDJ, 1950, t. 1, p. 77. 
Les droits culturels, comme les autres droits consacrés dans le texte de 1948, ont donc un fondement individualiste. Ce soubassement a précisément fait l'objet de critiques, l'individualisme étant étranger aux sociétés holistes, comme certaines sociétés africaines et asiatiques. Aussi, pour que les droits culturels soient susceptibles d'être exercés par tous, ils ont subi différentes inflexions : dans la droite ligne de la problématique propre aux droits des minorités, ils ont d'abord été appréhendés comme des droits individuels susceptibles d'être exercés collectivement; dernièrement, ils ont également été conçus comme les droits des «communautés culturelles».

\section{A. Des droits individuels aux droits collectifs}

Même si selon Gérard Cohen-Jonathan, «l'universalité de la Déclaration s'affirme également quant à ses destinataires: c'est l'être humain qu'elle entend protéger ${ }^{16}$, cette conception individualiste se heurte au fait que certains droits culturels n'ont de sens qu'exercés en commun avec d'autres. Autrement dit, certains droits culturels sont des droits collectifs ${ }^{17}$. Cet aspect n'est pas propre aux droits culturels; il concerne également des libertés, comme la liberté de réunion ou d'association et des droits économiques et sociaux : le droit de grève ou le droit syndical exercé par une unique personne n'aurait, par exemple, aucun sens.

Une telle dimension collective n'est pas non plus intrinsèque aux droits culturels: le droit de créer ou les droits d'auteur ont assurément un soubassement individualiste; si le caractère collectif concerne donc l'essentiel des droits des cultures, il n'est inhérent qu'à un faible nombre de droits à la culture. Étant donné l'importance croissante accordée aux premiers, un grand nombre de droits culturels possèdent aujourd'hui cette dimension. Ainsi, les droits linguistiques des membres d'un groupe, le droit d'organiser des manifestations culturelles traditionnelles, le droit de pratiquer des rites particuliers constituent des droits qui, par définition, se partagent et qui, à ce titre, doivent être exercés avec d'autres. Se pose alors un problème relatif à la nature des bénéficiaires de ces droits: s'agit-il des êtres humains membres d'un groupe ou s'agit-il du groupe lui-même?

Jusqu'à récemment, en dépit de l'évolution constatée, dans le sillage multiculturaliste, les droits culturels au sens anthropologique étaient plutôt conçus comme les droits des membres des collectivités ${ }^{18}$. En effet, si les multiculturalistes, au premier rang desquels Will Kymlicka ${ }^{19}$, estiment qu'un égal respect des communautés suppose la prise en considération des appartenances culturelles (au sens anthropologique) de chacun, ils restent attachés aux principes du libéralisme et, en particulier, à l'individualisme; ils entendent donc faire primer les droits individuels sur d'éventuels droits des groupes. Pour eux, la reconnaissance juridique des différences culturelles est envisagée "comme un moyen et une condition de la liberté ${ }^{20}$ : tout comme les droits économiques et sociaux dits de "seconde génération », les droits à l'identité culturelle doivent être appréhendés comme un nouvel enrichissement des droits individuels.

Les choses se compliquent toutefois lorsque l'on cherche à garantir ces droits. Ainsi, lorsque l'État les institutionnalise - en organisant par exemple des enseignements en langues régionales -, il prend nécessairement en compte des collectivités infra-étatiques ${ }^{21}$, donc des groupes. Et, le plus souvent, c'est parce qu'un individu fait partie d'une communauté minoritaire qu'il peut bénéficier de cet enseignement. D'ailleurs, après avoir tenté de répertorier les différents droits culturels ${ }^{22}$, le groupe de Fribourg note que «les droits culturels apparaissent entre les Droits de l'homme et les droits des peuples $»^{23}$.

16. G. Cohen-Jonathan, « Universalité et indivisibilité des Droits de l'homme», in La Déclaration universelle des Droits de l’homme, 1948-1998..., p. 45.

17. Sur cette question, voir notamment P. Texier, « Droits individuels et droits collectifs », in La Déclaration universelle des Droits de l'homme, 1948-1998..., p. $217 s q$.

18. On peut d'ailleurs s'étonner du rapprochement effectué dans la déclaration de Fribourg entre les personnes appartenant à une minorité et les personnes socialement défavorisées : l'article 9 dispose par exemple qu'il faut «veiller au respect des droits culturels, et développer des modes de concertation et de participation afin d'en assurer la réalisation, en particulier pour les personnes les plus défavorisées en raison de leur situation sociale ou de leur appartenance à une minorité ». Même si l'objectif est de veiller à ce que les plus démunis bénéficient de droits effectifs, faire le parallèle entre une situation précaire d'un point de vue social et une identité culturelle ne semble pas pertinent.

19. Voir notamment W. Kymlicka, La citoyenneté multiculturelle. Une théorie libérale du droit des minorités, Paris, La Découverte, 2001.

20. W. Kymlicka, «Les droits des minorités et le multiculturalisme», Comprendre. Revue de philosophie et de sciences sociales, W. Kymlicka et S. Mesure (dir.), $\mathrm{n}^{\circ}{ }_{1}$, Les identités culturelles, 2000, p. 148-149.

21. P. Vandernoot distingue, parmi les droits collectifs, «ceux qui ont un caractère proclamatoire à l'intention des minorités en tant que telles ou qui ont un caractère collectif par nature et ceux qui ont un caractère institutionnel et qui impliquent dès lors une organisation nécessairement collective de l'État». Il y ajoute " une troisième catégorie, située quelque peu à cheval entre les droits individuels et collectifs : il s'agit des droits reconnus éventuellement aux individus mais s'exerçant généralement en groupe ». P. Vandernoot, «Les aspects linguistiques du droit des minorités », RTDH, $\mathrm{n}^{\circ} 30$, $1^{\text {er }}$ avril 1997, p. 338. Notons que Geneviève Koubi qualifie ce dernier type de droits de « sociabilitaire » : selon elle, «l'enjeu de ces droits [...] est de conforter la dimension communautaire et identitaire des minorités [...] » (G. Koubi, « Droit et minorités dans la République française », in Le droit et les minorités. Analyses et textes, A. Fenet, G. Koubi et I. Schulte-Tenchkoff (dir.), Bruxelles, Bruylant, 1995, p. 208). Voir également G. Koubi, « Réflexions sur les distinctions entre droits individuels, droits collectifs et “droits de groupe” ", in Mélanges Raymond Goy, Rouen, Publications de l’Université de Rouen, 1998, p. 105-117.

22. Le droit à la participation culturelle (droit à la libre participation à la vie culturelle, droit au bénéfice du progrès scientifique et culturel, droit à la propriété intellectuelle), le droit à l'éducation (droit à l'éducation élémentaire et fonctionnelle, droit à l'orientation et à la formation professionnelle) et le droit individuel et collectif à l'identification culturelle (droit au choix de sa culture, droit à l'héritage culturel, droit d'accès aux moyens de communication et d'expression). Voir P. Meyer-Bisch (dir.), Les droits culturels, une catégorie sous-développée de Droits de l'homme, Actes du $8^{\mathrm{e}}$ colloque interdisciplinaire sur les Droits de l'homme à l'Université de Fribourg, 28-30 novembre 1991, Fribourg, Éditions universitaires de Fribourg, 1992, p. 13.

23. P. Meyer-Bisch, «Les droits culturels forment-ils une catégorie spécifique de Droits de l’homme?», ibid., p. 23. 
La seule brèche admissible vis-à-vis du fondement individualiste des droits culturels était, il y a encore quelques années, qu'ils puissent être exercés collectivement; le fait qu'ils soient destinés à des groupes semble désormais être admis.

\section{B. Des droits collectifs aux droits des communautés culturelles}

Aujourd'hui, même dans les textes à portée universelle, c'est-à-dire non centrés sur la problématique minoritaire, les droits culturels sont intrinsèquement liés aux droits des minorités. Or, la DUDH n'évoque ni les minorités, ni les populations autochtones : les droits culturels qu'elle reconnaît sont envisagés indépendamment de la reconnaissance d'identités culturelles.

Norbert Rouland explique que «ce n'est pas un oubli. Pour les minorités, on considère que leur manipulation a été une des causes qui ont engendré le conflit: il ne vaut donc mieux pas leur consacrer un traitement spécifique. Pour les autochtones, on pense que le développement économique va les intégrer aux sociétés dominantes. Dans les deux cas, on juge qu'une pleine et entière application des Droits de l'homme conçus dans un sens universaliste est nécessaire et suffisante pour régler les problèmes de ces groupes ${ }^{24}$.

La réalité était un peu plus complexe: les différents États étant très partagés quant à la nécessité d'une disposition relative à cette question ${ }^{25}$, un article protégeant les personnes relevant de groupes minoritaires a été introduit à plusieurs reprises. L'Union soviétique défendait notamment la disposition suivante : "Chacun a droit à sa propre culture ethnique ou nationale [...] ; à la création de ses propres écoles et à l'enseignement de sa propre langue, ainsi qu'à l'emploi de cette langue dans la presse, les réunions publiques, les tribunaux et les autres institutions de l'administration ${ }^{26}$.»

Il semblerait donc que ce soit plutôt l'absence de consensus sur une telle disposition, liée à une variété des situations, qui ait empêché l'approbation d'un tel article. Une résolution renvoyant au Conseil économique et social les textes relatifs aux minorités fut alors adoptée.

Ces hésitations originelles montrent que le problème des destinataires des droits des minorités n'est pas nouveau et se posait dans les mêmes termes il y a soixante ans. Et si, rapidement, les droits culturels ont été reliés aux droits des membres des minorités, de façon plus furtive, ils ont aussi été adossés à des droits des groupes. Ainsi, Janusz Symonides rappelle-t-il que « La Déclaration de Mexico [de 1982] sur les politiques culturelles spécifie notamment que l'affirmation de l'identité culturelle contribue à la libération des peuples ${ }^{27}$. Dans les textes les plus récents, des groupes - peuples autochtones ou communautés culturelles - sont explicitement considérés comme bénéficiaires de droits culturels. Il est à ce titre intéressant de constater que si les textes attribuent en général ces droits aux membres des minorités (donc à des individus), ils n'hésitent plus à en faire bénéficier directement les peuples autochtones (donc des collectivités). Ainsi, par exemple, la Déclaration universelle sur la diversité culturelle évoque-t-elle, à son article 4, «en particulier les droits des personnes appartenant à des minorités et ceux des peuples autochtones ». De même, dans le document explicitant la Convention de 2005, l'Unesco indique que les bénéficiaires du texte sont certes les individus, les professionnels et praticiens de la culture mais aussi «les divers groupes sociaux, y compris les femmes et les personnes appartenant aux minorités et les peuples autochtones ${ }^{28}$.

Il est permis de s'étonner du rôle joué par la France dans l'élaboration de ce texte, lorsque l'on sait qu'elle a coutume de formuler des réserves dès qu'une convention internationale consacre les droits des minorités. Ainsi, l'adhésion de la France au Pacte international relatif aux droits civils et politiques de 1966 par la loi du 25 juin 1980 a-t-elle été assortie d'une déclaration interprétative relative à l'article 27 du pacte international précisant que «compte tenu de l'article 2 de la Constitution [... l'article 27 n'a pas lieu de s'appliquer en ce qui concerne la République». La France a par ailleurs opposé une réserve à l'article 30 de la Convention relative aux Droits de l'enfant conclue dans le cadre de l'ONU et a, pour les mêmes raisons, refusé de signer la Convention-cadre pour la protection des minorités nationales adoptée par le Comité des ministres du Conseil de l'Europe le 10 novembre 1994. D'ailleurs, le Conseil constitutionnel a affirmé, dans sa décision nº 99-412 DC du 15 juin 1999 relative à la ratification de la Charte européenne des langues régionales ou minoritaires, que les principes figurant à l'article $1^{\text {er }}$ de la Constitution ainsi que «le principe d'unicité du peuple français dont aucune section ne peut s'attribuer l'exercice de la souveraineté nationale »-qui a également valeur constitutionnelle - «s'opposent à ce que soient reconnus des droits collectifs à quelque groupe que ce soit, défini par une communauté d'origine, de culture, de langue ou de croyance ${ }^{29}$; ils ont estimé « qu'il résulte de ces dispositions combinées que la Charte européenne des langues régionales ou minoritaires, en ce qu'elle conferre des droits spécifiques à des "groupes" de locuteurs de langues régionales ou minoritaires, à l'intérieur de "territoires" dans lesquels ces langues sont pratiquées, porte atteinte aux principes constitutionnels d'indivisibilité de

24. N. Rouland, «L'émergence du droit des minorités et des peuples autochtones dans les conventions et traités internationaux », in Le droit à la différence, N. Rouland (dir.), Aix-en-Provence, Presses universitaires d'Aix-Marseille, 2002, p. 193.

25. Sur les débats relatifs à l'insertion d'une disposition sur les minorités dans la Déclaration, voir W. A. Schabas, «Les droits des minorités : une déclaration inachevée ", in La Déclaration universelle des Droits de l'homme, 1948-1998..., p. 223 sq.

26. Ibid.

27. J. Symonides, «Les droits culturels : une catégorie négligée de Droits de l’homme », p. 620.

28. Voir note 10.

29. Décision n 99-412 DC du 15 juin 1999 relative à la ratification de la Charte européenne des langues régionales ou minoritaires, cons. 6. 
la République, d'égalité devant la loi et d'unicité du peuple français $»^{30}$.

Or, la Convention de l'Unesco de 2005, largement soutenue par la France, se réfere explicitement aux droits des peuples autochtones. Pour preuve, l'article $2 \S 5$ dispose que « la culture étant un des ressorts fondamentaux du développement, les aspects culturels du développement sont aussi importants que ses aspects économiques, et les individus et les peuples ont le droit fondamental d'y participer et d'en jouir ${ }^{31}$.

De même, l'article 7 indique que « les parties s'efforcent de créer sur leur territoire un environnement encourageant les individus et les groupes sociaux: a. à créer, produire, diffuser leurs propres expressions culturelles et à $y$ avoir accès $[\ldots]{ }^{32}$.

Un nouveau pas semble avoir été franchi avec la Déclaration de Fribourg de septembre 2007, laquelle consacre explicitement les " communautés culturelles ", définies comme des «groupe(s) de personnes qui partagent des références constitutives d'une identité culturelle ${ }^{33}$ commune, qu'elles entendent préserver et développer». Si l'article 4 a dispose que «toute personne a la liberté de choisir de se référer ou non à une ou plusieurs communautés culturelles, sans considération de frontières, et de modifier ce choix » et maintient donc le référentiel individualiste, l'article 8 indique notamment que «toute personne, seule ou en commun, a droit de participer selon des procédures démocratiques : au développement culturel des communautés dont elle est membre », consacrant ainsi implicitement un droit pour les communautés au développement culturel.

Une telle reconnaissance ne constitue plus un cas isolé : le 13 septembre 2007, a été adoptée la Déclaration des Nations unies sur les droits des peuples autochtones. Sans détour, elle accorde des droits directement aux peuples autochtones, donc à des groupes. Ainsi, l'article 3 dispose que «Les peuples autochtones ont le droit à l'autodétermination. En vertu de ce droit, ils déterminent librement leur statut politique et assurent librement leur développement économique, social et culturel ».

Innovant est également l'article $11 \S 2$, selon lequel «Les États doivent accorder réparation par le biais de mécanismes efficaces - qui peuvent comprendre la restitution - mis au point en concertation avec les peuples autochtones, en ce qui concerne les biens culturels, intellectuels, religieux et spirituels qui leur ont été pris sans leur consentement préalable, donné librement et en connaissance de cause, ou en violation de leurs lois, traditions et coutume».

Garantir des droits culturels directement à des communautés culturelles permet de rompre avec une conception occidentale de la notion de culture, d'une part, des bénéficiaires des droits, d'autre part. Toutefois, une telle affirmation comporte aussi le risque d'un relativisme culturel et moral légitimant des pratiques culturelles contraires aux Droits de l'homme. Conscients de ces potentielles dérives, les rédacteurs des textes internationaux ont souhaité les anticiper grâce à l'affirmation d'une hiérarchie entre Droits de l'homme et droits culturels. Ainsi, aux termes de l'article 5 de la Déclaration universelle sur la diversité culturelle, «Toute personne doit pouvoir participer à la vie culturelle de son choix et exercer ses propres pratiques culturelles, dans les limites qu'impose le respect des Droits de l'homme et des libertés fondamentales».

De même, l'article 2 alinéa $1^{\text {er }}$ de la Convention de l'Unesco sur la protection et la promotion de la diversité des expressions culturelles dispose que «Nul ne peut invoquer les dispositions de la présente Convention pour porter atteinte aux Droits de l'homme et aux libertés fondamentales tels que consacrés par la Déclaration universelle des Droits de l'homme ou garantis par le droit international, ou pour en limiter la portée ».

Bien que cette hiérarchisation puisse être conçue comme une garantie de l'universalisme des Droits de l'homme, il est permis de se demander si elle n'entre pas en contradiction avec leur indivisibilité, principe en vertu duquel les Droits de l'homme, quels qu'ils soient, sont d'égale valeur.

Indépendamment de la primauté donnée aux Droits de l'homme, il semblerait important de concevoir des droits culturels indépendamment des identités culturelles afin de ne pas oublier que la culture peut être conçue de façon autonome, sans lien avec un groupe particulier, et qu'elle peut être partagée par des individus relevant de communautés différentes. À force de vouloir garantir un droit à la différence culturelle, on risque en effet de faire primer ce qui distingue sur ce qui est susceptible de rassembler. L'article 7 de la Déclaration de Fribourg illustre une telle logique: "Dans le cadre général du droit à la liberté d'expression, y compris artistique, des libertés d'opinion et d'information, et du respect de la diversité culturelle, toute personne, seule ou en commun, a droit à une information libre et pluraliste qui contribue au plein développement de son identité culturelle [...].»

On peut alors se demander s'il est indispensable d'évoquer l'« identité culturelle» lorsqu'il s'agit de garantir la liberté d'expression.

Par ailleurs, l'évolution sémantique précédemment évoquée n'a été accompagnée d'aucune distinction entre sphère publique et sphère privée. Bien qu'occidentale, une telle différenciation a le mérite de permettre l'épanouissement des droits culturels anthropologiques tout en garantissant un lien entre les citoyens dans la sphère publique en en maintenant l'unité. Or, au regard des récentes évolutions, il est permis de penser que la reconnaissance

30. Décision n 99-412 DC du 15 juin 1999 relative à la ratification de la Charte européenne des langues régionales ou minoritaires, cons. 10. Nous soulignons.

31. Nous soulignons.

32. Nous soulignons.

33. L’identité culturelle étant elle-même définie comme «l'ensemble des références culturelles par lequel une personne, seule ou en commun, se définit, se constitue, communique et entend être reconnue dans sa dignité » (art. 2). 
ambiguë des identités culturelles risque d'être comprise comme une consécration d'une pluralité d'identités politiques. Ce serait alors la consécration de la logique communautarienne, illustrée notamment par la «politique de la reconnaissance» de Charles Taylor, laquelle ne se conçoit pas sans l'octroi aux communautés culturelles de droits d'une valeur au moins équivalente à celle des libertés individuelles ${ }^{34}$.

Pour Hector Gros Espiell, « un relativisme culturel radical et absolu, qui nierait la possibilité qu'existe le moindre élément commun et universel dans la culture humaine contemporaine, et qui [...] impliquerait la négation de caractère général des Droits de l'homme et de ses résultantes éthiques, politiques et religieuses serait incompatible avec l'idée d'universalité de ces droits et avec toute perspective d'une conception commune. En revanche, un relativisme culturel normal, rationnel, équilibré et moderne, fondé sur le constat de la réalité de particularités et de diversités historiques, religieuses et traditionnelles, régionales et nationales [...] se trouve à la base du nécessaire et juste équilibre entre universalité, diversité, homogénéité et différence, lequel doit être le point d'appui, équitable et réaliste, de la reconnaissance, de la promotion et de la protection internationale des droits de la personne humaine ${ }^{35}$.

Un des enjeux majeurs du XXI ${ }^{\mathrm{e}}$ siècle consiste ainsi à déterminer jusqu'où on peut aller pour «enrichir l'universalité par la diversité ${ }^{36}$.

À notre sens, l'universalisme des droits culturels ne pourra être effectif qu'au terme d'une double clarification. Afin que les droits culturels puissent être garantis en dehors de la problématique identitaire, les droits à la culture doivent impérativement être défendus indépendamment des droits des cultures. Par ailleurs, pour rompre avec la conception ethnocentriste, les droits des cultures doivent également être reconnus, en veillant à les accorder à des individus, non à des groupes : les individus doivent rester libres, non seulement de pratiquer ou de ne pas pratiquer tel droit culturel, mais encore de changer de groupe d'appartenance.

34. C. Taylor, «L'atomisme», La liberté des modernes, Paris, PUF, 1997, p. 224-225. Pour les communautariens, non seulement l'appartenance à un groupe fait naître des droits particuliers au profit de ses membres, mais encore les groupes doivent, en tant que tels, posséder des droits. Les communautariens entendent en effet protéger les communautés culturelles contre trois menaces potentielles : celles des individus, celles des autres communautés et celles de l'État. Tout doit être mis en œuvre pour préserver les différentes collectivités d'appartenance.

35. H. Gros Espiell, «Universalité des Droits de l’homme et diversité culturelle », Revue internationale des sciences sociales, $\mathrm{n}^{\circ} 158$, décembre 1998 , p. 591.

36. Article 9 de la Déclaration de Fribourg. 
